\title{
Modelización del análisis de las desigualdades en la distribución de la renta
}

\author{
Manuel Garcta Docampo \\ Universidade da Coruña
}

\section{OBJETO DE ESTUDIO}

El objetivo genérico de este artículo es el proporcionar un modelo analítico de las desigualdades en la distribución de la renta. Tal modelización se hace siguiendo un esquema secuencial que parte de una redefinición conceptual de las desigualdades sociales para, seguidamente, distinguir los diferentes tipos de situaciones discernibles sobre ellas. Precisamente uno de esos tipos es el de las distribucionales. Estas últimas pueden entenderse como una imputación entre dos conjuntos, el poblacional y el de los bienes. La asignación diferencial de los elementos del conjunto de los bienes sobre el de los sujetos mantiene una serie de reglas, cuyas transferencias implican procesos de equidistribución o de concentración. La renta es una de las variables susceptible de análisis desigualitario de tipo distribucional, tal como aquí aparece caracterizado y cuyos instrumentos métricos se exponen desde una perspectiva crítica y pedagógica.

Las desigualdades existentes en la distribución de la renta pueden medirse con tres tipos de indicadores. Por un lado, empleando toda una serie de medidas de tendencia central o de dispersión; por otro, calculando índices que valoren la concentración del total de renta existente, bien teniendo en cuenta únicamente las unidades existentes o bien considerando los incrementos del nivel de bienestar general de la población. Finalmente, también se puede partir de la definición de una ley probabilística que resuma la forma completa de la distribución.

Este último tipo de modelo analítico mantiene todo el potencial descriptivo del que gozan los anteriores, siendo posible el cálculo de los diferentes momentos de la distribución, así como de los índices de concentración, además de permitir extrapolaciones predictivas. Es posible constatar empíricamente que, en concreto, tres tipos de leyes teóricas se ajustan bien a la distribución de la renta 
observada: la distribución Log-normal, la Exponencial y la de Pareto. Su constatación empírica se hace calculando el coeficiente de determinación para siete distribuciones de la renta, cada una de ellas elaborada por organismos diferentes y sobre la base de diferentes conjuntos, tanto de bienes como de sujetos.

La línea expositiva sigue la lógica secuencial expuesta y permite una doble lectura. Por una lado, es posible seguir el argumento de modelización del análisis de las desigualdades de la renta a partir de un marco teórico concreto. Por otro lado, para el lector más interesado en el conocimiento estadístico-instrumental, se incluyen las herramientas métricas y, sobre todo, un anexo final en el que se indica, con cierto grado de detalle, el procedimiento de ajuste de esas tres leyes teóricas a las distribuciones observadas.

\section{EL MARCO TEÓRICO DE LA IGUALDAD}

Definir lo que entendemos por igualdad (o su antónimo, desigualdad) es una tarea compleja y requiere un ámbito expositivo más amplio del aquí dispuesto ${ }^{1}$. Sin embargo, es necesario aclarar algunos aspectos esenciales del marco teórico y del concepto de igualdad en particular.

Dada la imprecisión terminológica que la palabra igualdad impone en nuestro idioma y la polisemia que lleva asociada, encontramos, en la literatura al respecto e incluso en el leguaje común, una enorme diversidad de definiciones ${ }^{2}$ que pueden conllevar prejuicios poco útiles para un análisis riguroso. El concepto de igualdad se emplea en ámbitos científicos dispares y en sentidos de los que es difícil extraer un denominador común. Un geógrafo puede hablar de una superficie desigual, para referirse a la irregularidad del terreno; un matemático se refiere a una desigualdad cuando entre los dos miembros de una función o ecuación puede establecerse una relación de «mayor que» o «menor que»; un demógrafo habla de desigualdades para referirse a estructuras demográficas desequilibradas; un economista entiende que hay igualdad cuando dos personas poseen la misma cantidad de rentas; los juristas emplean usualmente el término en el sentido de estar en posesión de los mismos derechos; entre los sociólogos podemos contar con una ingente literatura sobre las desigualdades y en una no menos numerosa cantidad de significados: oportunidades, trato, capacidades, bienestar, estratificación social, prestigio, etc ${ }^{3}$.

1 Podemos distinguir, cuando menos, los siguientes factores incidentes en dicha complejidad: la imprecisión terminológica; la inabarcable producción literaria que al respecto hemos ido acumulando a lo largo de la historia; los innumerables contextos histórico-culturales en los que se definen problemáticas de desigualdades sociales; la aproximación multidisciplinar requerida para su análisis; la magnitud analítica posible; la multiplicidad de ámbitos sociales susceptibles de ser desigualitarios; la imprecisión de la Sociología de las desigualdades; las connotaciones político-morales que encierra.

2 Rae habla en su Equalities de 720 tipos de significados de la idea de desigualdad (Rae, 1981:189), aunque en realidad está incluyendo analíticas muy diferentes: estudio conceptual, medida, actitudes ante la igualdad, políticas igualitaristas, captación de situaciones desigualitarias, etc., entre otras.

3 En sociología habría que incluir la polémica sobre el carácter heurístico de las teorías de clases sociales, así como su centralidad explicativa del resto de desigualdades (Crompton, 1993:17-40). 
A partir de los numerosos ejemplos con los que podríamos ilustrar la polisemia conceptual asociada ${ }^{4}$, debemos plantearnos si es posible sacar algún denominador común entre todas ellas y preguntarnos si es viable una definición de igualdad social. En realidad, sí es factible si partimos de una serie de supuestos cuya existencia conjunta permitirá un tipo concreto de analítica que podemos denominar como igualitarista. Dichos supuestos deberán caracterizar las situaciones reales sobre las que se aplicará el análisis de forma que sean «sociales», «potencialmente igualitarias» y que estén «formuladas como una comparación situacional».

En el marco de la concepción político-moral existente en cada cultura determinada se definen aspiraciones y anhelos de organizarse socialmente en múltiples ámbitos. Se desea una determina «situación» de relación de unos seres humanos con los otros intentando salvar las diferencias producidas por la diversidad humana. Toda situación social, real o teórica, es susceptible de ser analizada en cuanto a su carácter igualitario, si en la configuración de la misma son discernibles tres elementos: 1) Ha de ser social, es decir, producto de una determinada forma de organización social; 2) Deben definirse previamente los supuestos de igualdad absoluta y desigualdad absoluta; 3) Deben estar presentes, en su formulación, los tres elementos siguientes: un conjunto poblacional de referencia, una variable evaluativa y un sistema o criterio de comparación (Rae, 1981: 106-7; Sen, 1992: 57-58). En función del tipo de comparación, de la naturaleza de la o las variables evaluativas y de las características del conjunto poblacional sobre el que formulemos una determinada situación potencialmente (des)igualitaria, podremos finalmente definir si existe o no (des)igualdad y la intensidad de la misma ${ }^{5}$. La evaluación de la comparación puede ser de tres tipos ${ }^{6}$ :

1. Podemos comparar una distribución de un determinado bien entre los elementos del conjunto en una situación dada. Llamaremos a este tipo de comparaciones Distribucionales o Imputacionales.

2. Podemos comparar la posibilidad de acceso entre los diferentes elementos del conjunto a una determinada situación. Llamémoslas Probabilidades de Paso.

${ }^{4}$ Es preciso mencionar la obra colectiva, compilada por Amelia Valcárcel, sobre el concepto de Igualdad (Valcárcel, 1994).

5 Se trata de una definición de igualdad de carácter axiomático, recurso empleado recurrentemente en ámbitos científicos diversos que van desde la Filosofía a las Matemáticas. En general. axiomas son aquellas afirmaciones que se presentan como premisas evidentes que no precisan de demostración. Su aceptación es arbitraria y consensual, respondiendo a una determinada estrategia que permita, a su vez, definir conceptos que son intrínsecamente arbitrarios. Un ejemplo de definición axiomática es la definición de las probabilidades. En estadística se intentó definir la probabilidad por medio de fórmulas tipo casos favorables entre casos posibles; ese tipo de definiciones resultó falso en la práctica, por lo que se recurrió a la clásica tríada de axiomas de la probabilidad.

${ }^{6}$ La razón por la que aparecen estos tres tipos de situaciones, aunque no profundicemos en ello ahora, viene dada por la combinación de los tipos de comparaciones posibles que podemos llevar a cabo (inter o intra) sobre el conjunto poblacional y el tipo de variables evaluativa (cuantitativa o cualitativa). No existen metodológicamente más posibilidades, si bien estas tres están a un nivel primario de desagregación; una descomposición posterior de cada una de ellas nos llevaría a distinguir entre diferentes tipos bajo cada uno de sus epígrafes. 
3. Podemos comparar la posesión de una determinada condición por los elementos del conjunto en una situación determinada. Nos referiremos a esta situación como Dicotomías Nominales.

Es sobre cada uno de esos tres tipos de formulaciones, y atendiendo a los postulados y características axiomáticas de la formulación, sobre los que puede definirse la existencia de una desigualdad social. Las situaciones distribucionales mantienen un extremo igualitario de equidistribución, frente a otro totalmente desigualitario de concentración total; las probabilidades de paso reflejan probabilidades diferentes entre los elementos del conjunto poblacional para conseguir y alcanzar determinada posición o situación; las dicotomías nominales ponen de manifiesto que algunos elementos están en posesión de una determinada característica ausente en el resto.

\section{LAS DESIGUALDADES DISTRIBUCIONALES}

\subsection{Definición de las desigualdades distribucionales}

Siempre que hablemos de desigualdad distribucionales precisamos dos conjuntos: el conjunto $\boldsymbol{B}$ de los bienes susceptibles de ser repartidos entre el conjunto $I$ de los individuos (ambos conjuntos finitos). Cada bien ${ }^{7}$ se atribuye a un individuo y sólo a uno; un individuo puede tener varios bienes, uno solo o eventualmente ninguno. La función que hemos definido se denomina imputación. Una imputación es una función de $\boldsymbol{B} \rightarrow \boldsymbol{I}$ en donde a cada bien se le hace corresponder un individuo que lo percibe. Es sobre estas imputaciones donde podemos hablar de desigualdad ${ }^{8}$. El término desigualdad, en su sentido de distribucional, estará estrechamente ligado al de concentración. Por situación de igualdad entendemos equidistribución; término opuesto al de concentración.

$\mathrm{El}$ conjunto de bienes tiene las siguientes características:

- Pueden ser bienes simples o complejos, entendidos, estos últimos, como una combinación de bienes simples.

- Los bienes pueden ser materiales, culturales o de cualquier otra naturaleza; por supuesto, se incluyen todo tipo de servicios.

- Los bienes deben ser perfectamente identificables.

- Los bienes deben ser asignables o asociables a todos los elementos del conjunto poblacional. Si bien en la práctica no tiene por que darse esa

${ }^{7}$ Ni que decir tiene que las imputaciones, al hablar de desigualdades sociales, han de ser de carácter social.

${ }^{8}$ Quizá haya sido Marc Barbut quien sintetizara por primera vez y de una forma más exhaustiva estas desigualdades socioeconómicas a las que nos referimos. Este autor propone una formalización en dos fases. En una primera se define el campo o ámbito matemático en el que se produce esa situación de igualdad o desigualdad. En una segunda fase se precisarían las reglas o propiedades que debe mantener una desigualdad para que sea considerada como tal. (Véase especialmente en Barbut, 1989). 
situación, substantivamente ha de ser perfectamente posible (potencialidad igualitaria).

- El conjunto de los bienes debe tener un número de elementos que oscilan entre dos y un número finito indeterminado

El conjunto poblacional no precisa ninguna particularidad. Puede ser un conjunto de individuos simple o una identidad de cualquier tipo (geográfica, cultural, social, política, fisiológica, económica, etc. ${ }^{9}$ ). La comparación se refiere al hecho de tener igual, más o menos unidades del conjunto de bienes definido. Este conjunto debe estar compuesto por una serie metodológicamente finita de unidades definibles, cuantificables y susceptibles de ser atribuibles o asignados (imputación) a todos y cada uno de los miembros del conjunto poblacional.

Dado que el ámbito sobre el que basar las desigualdades distribucionales es de naturaleza cuantitativa, podemos apoyar nuestro criterio comparativo en el estudio de la distribución de los elementos del conjunto de los Bienes de forma equidistributiva (la situación de igualdad absoluta vendría definida por la situación en la que todos los elementos del conjunto poblacional $I$ tienen el mismo número o cantidad de elementos del conjunto de los $B$ de los Bienes) frente a una distribución desigualitaria basada en la concentración (la situación de desigualdad absoluta se definiría en el caso de que un elemento del conjunto poblacional tiene todos los elementos del conjunto $B$, mientras que el resto de los miembros del conjunto $\boldsymbol{I}$ no poseen ningún elemento de $\boldsymbol{B}$ ). Entre esas dos situaciones extremas existen toda un serie de situaciones intermedias, que consideraremos como situaciones de desigualdad relativa. Nuestra medida de la intensidad de la desigualdad se basará en una referencia hacia la proximidad a una situación de igualdad absoluta o de desigualdad absoluta.

\subsection{Propiedades}

Existen una serie de características o reglas de las imputaciones definidas que nos ayudan a identificar las desigualdades sociales que estudian.

\section{Propiedad primera: Las particiones y sus redes.}

Dos imputaciones que se deducen una de la otra por una permutación de los elementos de $I$ tienen la misma desigualdad. O lo que es lo mismo: la desigualdad (y la concentración) de una imputación es invariante respecto al grupo de las permutaciones de $\mathbf{I}$. Poco importa quién es el individuo que recibe los bienes sino el número de subconjuntos de clases de bienes que se constituyen ${ }^{10}$.

${ }^{9}$ Como conjunto, mantiene las reglas de reflexividad, simetría y transitividad. Esto. que aparentemente puede parecer más que discutible en teoría, no lo es, como veremos, en la práctica desde nuestra definición de desigualdades sociales. La Simetría, el que todo elemento tenga el mismo valor para la asignación de una característica determinada es una condición necesaria en nuestra definición de situación potencialmente (des)igualitaria.

${ }^{10} \mathrm{Al}$ hablar de imputaciones es suficiente con referimos al conjunto origen. 
Se deduce de esta regla que toda transferencia de una parte de los bienes de un poseedor hacia un no-poseedor reduce la concentración y la desigualdad.

Segunda propiedad: Las distribuciones (reparticiones).

La desigualdad de una imputación es invariante respecto al grupo de permutaciones de $\boldsymbol{B}$. En este caso lo que cuenta no son los bienes atribuidos a cada individuo sino su cantidad.

Sea $I=\{1,2, \ldots, i, \ldots, k\}$. La distribución (o repartición) de una imputación es el vector .

$$
x=\left(x_{1}, x_{2}, \ldots, x_{i}, \ldots, x_{k}\right)
$$

en donde $\mathrm{x}_{\mathrm{i}}$ es el número de unidades de bienes atribuidas al individuo $i$, y la suma de las $x_{i}$ es igual a $n$, efectivo total del conjunto $B: \forall \mathrm{i}, \mathrm{x}_{\mathrm{j}} \geq 0 ; \sum x_{i}=n=|B|$.

Se puede demostrar con facilidad que el número de imputaciones admitiendo la misma distribución $\left(x_{1}, x_{2}, \ldots, x_{i}, \ldots, x_{k}\right)$ responde a la expresión llamada «multinomial»:

$$
\frac{n !}{x_{1} ! x_{2} ! \ldots x_{k} !}=\frac{\left(\sum x_{i}\right)}{\prod\left(x_{i} !\right)}
$$

\section{Tercera propiedad: Las reparticiones y sus curvas de concentración.}

Si tenemos en cuenta simultáneamente las propiedades $1^{\mathrm{a}}$ y $2^{\mathrm{a}}$, la desigualdad de una imputación $\boldsymbol{B} \rightarrow \boldsymbol{I}$ es invariable tanto con respecto al grupo de las permutaciones de $\boldsymbol{I}$ como con respecto al de $\boldsymbol{B}$. De esta manera no tendremos que quedarnos de cada imputación más que con el conjunto de partes de bienes recibidos por los individuos: la repartición del número $n=|\boldsymbol{B}|$ en $k=|\boldsymbol{I}|$ partes.

Es necesario ahora ordenar las reparticiones desde el punto de vista de las desigualdades en el sentido de «desigualdades de una repartición». Así, si una transferencia de una unidad se lleva a cabo de una parte $n_{i}$ a una parte $n_{j}$ inferior $\left(n_{i}>n_{j}\right)$, entonces, o bien no varía el reparto $\left(\operatorname{si} n_{i}-n_{j}=1\right)$, o bien $\left(\operatorname{si} n_{i}-n_{j}\right.$ $=1$ ) se reduce la desigualdad de la repartición. Iterando las transferencias de los poseedores hacia los no-poseedores se obtienen progresivamente reparticiones cada vez más igualitarias. Toda transferencia de unidad de un bien de una parte hacia una parte inferior reduce la desigualdad de la repartición (o eventualmente la deja invariante). Esta regla es conocida en la literatura socioeconómica con el nombre de Principio de Pigou y Dalton ".

${ }^{11}$ Mencionan esta regla las obras de Atkinson (1983) o Sen (1973). 


\section{Cuarta propiedad: Concentración normalizada.}

Si queremos hacer comparaciones entre diferentes imputaciones precisamos pasar todos nuestros datos (tanto los individuos como las partes que reciban) a proporciones. Esto nos permitirá normalizar y calcular indicadores autosuficientes, como el de Lorentz-Gini que más adelante veremos.

\subsection{Aplicación al estudio de la renta.}

Las variables evaluativas de una situación desigualitaria de tipo distribucional son muy diversas. Siempre que se cumplan los requisitos enunciados en su formulación axiomática, todas pueden incluirse. Dependiendo del tipo de conjunto poblacional $I$, podemos distinguir diversos tipos de distribución de la renta ${ }^{12}$ : a) distribución personal, es decir, distribución entre las distintas clases de personas que perciben ingresos o tienen un patrimonio individual o familiar; b) distribución funcional entre los factores del capital, del trabajo y de la tierra; c) distribución social, por profesiones, clases, capas sociales, sexo, distintas minorías, grupos de edad, etc.; d) distribución regional, por agregados territoriales o espaciales.

El matiz específico que queramos concederle a la definición de renta hará que el análisis sea de un tipo u otro. Generalmente las definiciones de renta dependen de las fuentes de datos existentes en cada sociedad y sus posibilidades de explotación; los investigadores no tienen más remedio que adaptar las definiciones a la información disponible. En general, las rentas personales pueden definirse globalmente o por indicadores parciales. Para hacerlo globalmente suele escogerse uno de estos tres criterios: I) los ingresos, incluidos salarios, rentas por patrimonio y todo tipo de inputs cuantificables monetariamente y, normalmente, tomando hogares; II) el gasto por hogar y por período, incluyendo seguridad social, impuestos, etc.; III) el montante resultante de la diferencia entre ingresos y gastos en un período determinado y en un hogar determinado, incluidas las rentas declaradas a nivel impositivo y fiscal ${ }^{13}$. Cuando se hace por medio de indicadores parciales suele utilizarse únicamente una o varias variables disponibles (Salario, Declaración del IRPF, etc.)

\section{INSTRUMENTOS DE MEDIDA}

\subsection{Indicadores basados en la tendencia central}

Las imputaciones así definidas identifican la existencia de desigualdades y la intensidad de la mismas, permitiendo comparaciones si nos servimos del bagaje

12 El historiador alemán Harmut Kaelble (1983) efectúa eșa distinción y la aplica en su análisis de la primera parte de su obra (pp. 21-246) aunque se encuentra un mención más específica a esa distinción en pp. 37 y 38.

13 Véase esta definición en Mercader, 1993, p. 9. 
estadístico analítico adecuado. Las imputaciones de bienes a los individuos constituyen una distribución de efectivos sobre los cuales podemos calcular una serie de momentos que servirán para resumirlas y caracterizarlas. Entre los indicadores basados en medidas de tendencia central y en su dispersión intrínseca, cabe destacar: la desviación media relativa $(M)$, la varianza $(V)$, el coeficiente de variación $(C)$ y la varianza de los logaritmos $(H)$.

La desviación media relativa $(M)$ considera a todos los valores de la distribución y compara el nivel de renta de cada individuo con la renta media, sumando los valores absolutos de todas las diferencias y considerando la suma como la proporción de la renta total:

$$
M=\sum_{i=1}^{n}\left|\mu-y_{i}\right| / n \mu
$$

Cuando la igualdad es perfecta, $M=0$ y cuando un solo individuo recibe toda la renta, $M=2(n-1) / n$.

El principal problema de la desviación media relativa es que no resulta sensible a las transferencias de renta de un individuo pobre a otro más rico cuando ambos se encuentren en el mismo lado con respecto a la renta media.

Si en lugar de sumar simplemente los valores absolutos, elevamos previamente dichas diferencias al cuadrado, acentuaremos las distancias más alejadas de la media, obteniendo la fórmula de la varianza habitual de la estadística.

$$
V=\frac{\sum_{i=1}^{n}\left(\mu-y_{i}\right)^{2}}{n}
$$

La varianza será sensible a cualquier transferencia de una unidad de bien de un individuo pobre a otro más rico y viceversa, siguiendo así el principio de Pigou-Dalton (propiedad tercera de las desigualdades basadas en distribuciones probabilísticas), pero sin informar sobre el sentido de la transferencia. Además, la varianza sigue dependiendo del nivel medio de la distribución y, en definitiva, de los valores absolutos que la configuran. Esto ha pretendido solventarse con el Coeficiente de Variación:

$$
C=\frac{\sqrt{V}}{\mu}
$$

Este coeficiente relativiza y soluciona el problema del valor de las medias, pero continúa siendo insensible al sentido de las transferencias de los poseedores de bienes.

La varianza de los logaritmos es más sensible a las transferencias de la renta que los índices anteriormente expuestos, ya que incrementa las diferencias entre las rentas bajas y las altas, $y$, entre ellas, elimina la tendencia a concentrar las 
pequeñas distancias entre las rentas bajas. Además, es fácil de calcular, es descomponible aditivamente (es decir, permite la cuantificación de la desigualdad atribuible a subconjuntos de la población total -Comunidades Autónomas, categorías socioeconómicas, etc.-) y resulta adecuado para hacerse una primera idea de la magnitud de la desigualdad. Su procedimiento de cálculo responde a la siguiente ecuación:

$$
H=\frac{\sum_{i=1}^{n}\left(\log \mu-\log y_{i}\right)^{2}}{n}
$$

Sin embargo, como las anteriores, necesita del arbitrario recuso a la elevación al cuadrado, insensibilizándose con respecto al sentido de las transferencias, que siguen refiriéndose al valor medio.

\subsection{El Índice de Gini y la Curva de Lorenz}

El índice de Gini ${ }^{14}$ proporciona una mejor medida de la igualdad con su concepto de la equidistribución total y la proximidad de cada distribución observada a la equidistribución máxima posible. En la notación de Sen (1973: 31).

$$
G=\frac{1}{2 n^{2} \mu} \sum_{i=1}^{n} \sum_{j=1}^{n}\left|y_{i}-y_{j}\right|
$$

en donde el número de personas es $n$ y $\mu$ es la media aritmética de los bienes, $y_{i}$ e $y_{j}$ son los bienes de las personas $i$ y $j$ is.

Este índice se puede considerar con relación a la curva de Lorenz ${ }^{16}$, según la cual los porcentajes de población ordenados de más pobres a más ricos se representan en el eje horizontal y los porcentajes de renta disfrutados por el correspondiente porcentaje acumalado de la población se representan en el eje horizontal. Con el primer cuadrante de un eje de coordenadas podemos construir un cuadrado que tenga por lados los ejes de abscisas (en donde se representarán los porcentajes de población considerados-acumulados-) y el de ordenadas, (con el

14 Este índice se atribuye a Gini y fue extensamente analizado por Atkinson (1983), entre otros.

15 Para distribuciones de variable continua, podemos notar la fórmula del índice de Gini como:

$$
G=\frac{\Delta}{2 \mu}=2 \int_{0}\left[\int_{0} F(y)-L(y)\right] d F(y)=\frac{2}{\mu} \int_{0}^{\infty} y\left[F(y)-\frac{1}{2}\right] d F(y)
$$

${ }^{16}$ La exposición del autor se encuentra en Lorenz (1905). En Atkinson (1981 y 1983) y en Sen (1973) puede encontrarse una referencia a la amplia bibliografía existente sobre este instrumento métrico. 
porcentaje de bienes poseídos por esa población de referencia - por tanto, acumulada-). Si trazamos una línea continua entre los puntos obtenidos por los valores observados en dichas coordenadas, obtendremos una curva que irá de un extremo al otro del cuadrado, alejándose de la diagonal en el caso de concentración de los bienes o aproximándose a ella en el caso de equidistribución de los mismos.

\section{Cuadro n..$^{\circ}$ 1. Curva de Lorenz. Ingresos por hogar en España}

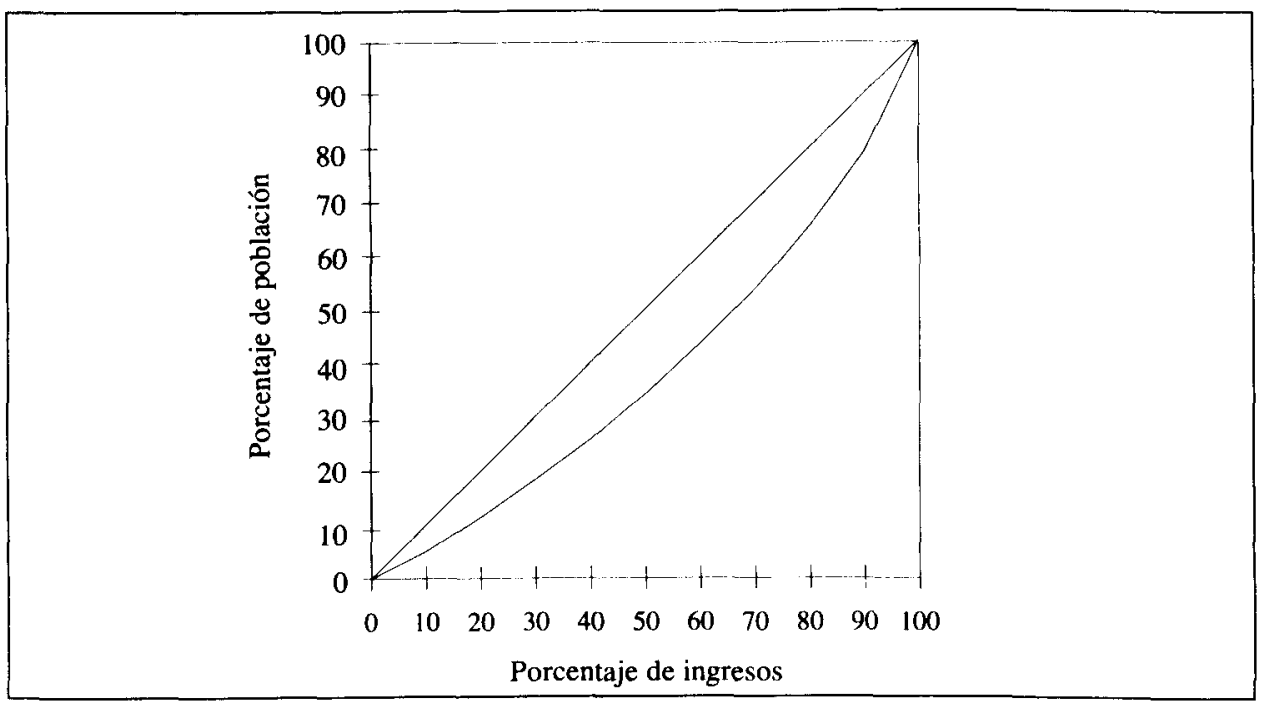

Fuente: Elaboración propia a partir de datos de la Encuesta de Presupuestos familiares 1990/1991. Principales Resultados. INE

El índice de Gini de una serie positiva cualquiera es el doble del área de la superficie comprendida entre la diagonal del cuadro (Bienes - Recursos, en porcentajes) y la curva de Lorenz de esa serie estadística. El índice de concentración de Gini de una serie estadística es un número comprendido entre 0 y 1 , puesto que el área del triángulo situado bajo la diagonal vale 0'5. Si el valor del Índice de Gini resultante es próximo a 0 , la curva de Lorenz está próxima a la diagonal, es decir, que la serie estadística está poco concentrada. Por contra, si este número es próximo a 1, significa que la curva de Lorenz se mantiene mayormente próxima al eje horizontal, lo que quiere decir que la serie estadística es muy concentrada.

Para calcular el área comprendida entre la diagonal y la curva de Lorenz, deben hallarse primero las áreas de los trapecios situados entre la curva y el eje horizontal y limitados por las verticales de los vértices de esa curva. (El primer trapecio es, por otra parte, un triángulo -recordamos que el área de un trapecio es igual al producto de su altura por la semisuma de las bases). Tomaremos después su complementario a 5.000 , si nuestros datos están expresados en porcentaje, y bastará con dividir por 5.000 el resultado obtenido. 
Sea una distribución en la que se dispone de dos conjuntos, $P_{i}$ y $Q_{i}$, ambos en porcentajes acumulados. Precisamos ordenar y calcular las siguientes columnas (A) y (B), según el formato que se indica a continuación:

\section{Cuadro n. ${ }^{\circ} 2$}

Formato de cuadro para el cálculo del índice empírico de Gini

\begin{tabular}{|c|c|c|}
\hline Columna (A) & Columna (B) & Columna (C) \\
\hline$P_{i}-P_{i-1}$ & $\frac{Q_{i}+Q_{i-1}}{2}$ & (A) $\times(\mathrm{B})$ \\
\hline
\end{tabular}

A continuación será preciso multiplicar cada elemento de la columna (A) por su correspondiente de la columna (B). Obtendremos así una nueva columna, a la que llamaremos (C). La diferencia entre 5.000 y la adición de los valores de (C) se dividirá por 5.000, para obtener finalmente el Índice de Gini de los valores observados.

A pesar de la estimable síntesis que este índice proporciona sobre el carácter igualitario de una distribución de bienes, ocasiona una deficiente visión de las distribuciones al no tener en cuenta que suele ser deseable una igualdad a partir de ciertos niveles de renta. Así por ejemplo, en la tabla siguiente, dos personas no tienen nada y luego, en una segunda situación, esas dos personas han mejorado su nivel de rentas, ¿Cuál de las dos situaciones es preferible?:

Cuadro n..$^{\circ} 3$

Disyuntiva de situación desigualitaria con incremento bienestarista

\begin{tabular}{|c|c|c|}
\hline & \multicolumn{2}{|c|}{ Situaciones } \\
\hline & I & II \\
\hline Sujeto $\mathrm{i}$ & 1 & 150 \\
\hline Sujeto $\mathrm{j}$ & 1 & 50 \\
\hline
\end{tabular}

El índice de Gini daría equidistribución perfecta, $\mathrm{G}=0$, en la primera situación y un valor representativo de una distribución mucho más desigualitaria en el segundo caso. Sin embargo, el sujeto $j$ probablemente preferirá su situación desfavorable, en términos desigualitarios, a la igualdad en la pobreza de la primera solución.

En definitiva, el índice de Gini, aunque sensible al principio de Pigou-Dalton, depende excesivamente del número de sujetos que se están midiendo. Los mercados y demás conjuntos poblacionales sobre los que habitualmente se aplica, no son cerrados. Entran y salen sujetos a formar parte del conjunto de referencia, incidiendo directamente en el valor del índice, lo que conlleva que haya que tomarlo con precauciones para realizar comparaciones tanto históricas, como 
entre sociedades diferentes ${ }^{17}$. En todo caso, puede considerarse como un índice que mide correctamente la igualdad en sentido estricto, sin tener en cuenta el bienestar colectivo que genera la misma.

\subsection{La igualdad bienestarista}

En cierto sentido el índice de Theil supera algunos de los problemas que en ocasiones puede conllevar la utilización del índice de Gini. Las ventajas que se le suponen al de Theil son de dos tipos: por un lado, supera el problema de las distancias entre los diferentes niveles de renta, al relativizar las proporciones de bienes o renta poseídos, gracias a la utilización de logaritmo de dichas proporciones; por otro, puede calcularse sin necesidad de partir de la ordenación creciente de los datos, como precisaba el de Gini. En cualquier caso, y en general, los resultados de aplicar uno u otro no suelen diferenciarse en exceso y sus valores suelen ser muy próximos.

Dada una población de $n$ sujetos, cada uno de los cuales posee una proporción determinada de bienes $x_{\mathrm{i}}$, el índice de Theil $(\mathrm{T})$, podría calcularse por medio de la expresión ${ }^{18}$ :

$$
T=\frac{1}{\ln (n)} \sum_{i=1}^{n} x_{i} \ln \left(\frac{x_{i}}{x}\right)
$$

En esta fórmula $x_{i}$ representa el número de sujetos que poseen la característica en cada una de las $i$ categorías investigadas (que puede venir dado en proporciones); $x$ es el número total de sujetos (poseedores o carentes del bien $o$ característica) y $n$ es el número de categorías investigadas. Al estar dividido por $\ln (n)$, el índice está normalizado a 1 .

Aunque el origen y la formulación de este índice ha sido tildada de arbitraria, en realidad mantiene sus raíces teóricas en la teoría de la información y su capacidad de medición de las desigualdades es inestimable (Theil, 1967). Con todo, es insensible a los incrementos totales de renta del conjunto de la población, algo que

17 Dado que las estructuras sociodemográficas son diferentes en Galicia y en Cataluña, éstas condicionarán estructuralmente el valor del índice de Gini resultante. Amartya Sen, consciente de este efecto, lo explica con el siguiente ejemplo: «Si A recibe un renta de 2.000 libras y B de 1.900 libras, y si A es el milésimo individuo más rico, mientras que B es el 1.100-ésimo individuo más rico, la entrega de 1 libra a B se considera equivalente a la entrega de l'10 libras a A. Pero si aparecen otros individuos dentro de este intervalo de renta, por ejemplo, si 100 personas más obtienen rentas comprendidas entre 1.900 y 2.000 libras, el coeficiente de Gini asignará la misma ponderación a la entrega de 1 libra de B que a la entrega de 1,20 libras a A» (Sen, 1973: 48-49). Por tanto, aunque los niveles de renta de A y B ha permanecido invariantes, sus ponderaciones sí han variado.

18 Así descrita, la fórmula sólo precisa tener en cuenta el número de efectivos, $n$ y los valores asociados a cada sujeto: $x_{i}$. Una aplicación empírica de los análisis desigualitarios basados en este índice puede encontrarse en Garrido/Toharia, 1996 (Los autores estudian la incidencia del desempleo en la sociedad española). 
había intentado controlar tempranamente Dalton (1920) y, sobre todo, posteriormente, Atkinson ${ }^{19}$, al construir un índice que calcula «la proporción de renta que sería necesaria para obtener una distribución igualitaria de la misma, sin pérdidas en el nivel actual de bienestar del conjunto poblacional» (Atkinson, 1981: 69). La fórmula para calcularlo puede expresarse del siguiente modo:

$$
A=1-\left[\sum_{i=1}^{n}\left(\frac{y_{i}}{y}\right)^{1-\varepsilon} f_{i}\right]^{\frac{1}{1-\varepsilon}}
$$

En ella se consideran los valores de las rentas $y_{i}$ de la población, en cada intervalo $(i)$, la renta media del conjunto $(\bar{y})$ y la proporción de población cuya renta se encuentra dentro del intervalo i-ésimo $\left(f_{i}\right)$. Además, se precisa, a priori, la estimación de un parámetro $\varepsilon$ que representa la ponderación asignada por la sociedad a la desigualdad. Éste variará desde 0 , en el caso de indiferencia total, hasta el infinito, en el caso de que las desigualdades fuesen la preocupación prioritaria de la población. La estimación de este último parámetro le confiere una carga de subjetividad muy difícil de consensuar en la práctica.

\section{LOS MODELOS TEÓRICOS}

Los índices presentados anteriormente proporcionan una visión excesivamente sintética de las características de la distribución de las rentas. Para obtener una información más amplia podemos recurrir a caracterizar la distribución observada por medio de un modelo probabilístico teórico, que se ajuste bien a los datos empíricos. De esta forma, podemos realizar una descripción más completa de la forma e intensidad de la desigualdad observada, ampliando, además, las posibilidades analíticas con la capacidad predictiva que proporcionan estos modelos. Se trata de un recurso habitual en las Ciencias Sociales que pretende

19 El Índice de Gini ha sido en el pasado la medida de desigualdad más comúnmente adoptada. Sin embargo, el posterior desarrollo de la teoría económica del bienestar ha dado lugar a un conjunto de propiedades normativas (S-convexidad, homoteticidad distributiva, etc.) que. junto con otras características (sencillez de cálculo, posibilidad de descomponerlo aditivamente, etc.) determinan los méritos relativos de los distintos índices de desigualdad que se han definido, de los que conviene destacar los basados en la varianza de los logaritmos (fáciles de calcular y descomponibles aditivamente, permitiendo la cuantificación de la desigualdad atribuible a subconjuntos de la población total -Comunidades Autónomas, categorías socioeconómicas, etc.); los índices de la familia de Theil (para el valor $\mathrm{c}=0$ del parámetro, se obtiene un índice que satisface las principales propiedades normativas $y$, además, es aditivamente descomponible de forma satisfactoria) y los de la familia de Atkinson (se caracterizan por medir la desigualdad en términos del coste potencial en el bienestar que ocasiona, incorporando explícitamente un conjunto de juicios de valor desde el principio del análisis, materializados en el valor del parámetro c. Aunque algún miembro es equivalente a $T_{0}$ en cuanto a satisfacción de propiedades normativas, su principal inconveniente radica en no ser aditivamente descomponible, lo que constituye un inconveniente grave cuando interesa repartir la desigualdad de la población total según determinados subgrupos) (INE, 1989: 24-25). 
resumir una realidad compleja en una serie de parámetros que, en definitiva, representan diferentes momentos de la distribución, de entre los que son especialmente relevantes las medidas de tendencia central y las de dispersión. Sin embargo, con frecuencia, se ha hecho un uso abusivo de la Distribución Normal como modelo teórico de la distribución de las variables de naturaleza sociológica. En el caso de la renta no es éste el modelo probabilístico más ajustado y, como veremos, otras leyes son susceptibles de ser definidas como representativas de las distribución empíricamente observada. En el caso que nos ocupa, las más usuales y que mejor se ajustan son las de Pareto, Log-normal y Exponencial. Las tres tienen una forma similar $y$, básicamente, se diferencian en el crecimiento más o menos intenso de las rentas más bajas y en el decrecimiento más o menos ralentizado de las rentas medio-altas y altas.

La bondad del ajuste ${ }^{20}$ de un modelo teórico ${ }^{21}$ a una distribución observada puede evaluarse calculando el coeficiente de determinación entre los datos observados empíricamente y el modelo teórico. En el cuadro siguiente $\left(n^{\circ} 4\right)$ se resumen los valores alcanzados por ese coeficiente para una serie de fuentes diferentes que proporcionan información sobre la distribución de la Renta en España y, en algún caso, en la Comunidad Autónoma de Galicia. Las tres distribuciones expuestas muestran una muy buena correlación, con valores de $r^{2}$ por encima del 0,8 . De esto se deduce que independientemente de cuál sea la fuente, el año y el tipo de renta recogido, se trata siempre de un tipo de distribuciones bien definidas por cualquiera de las tres leyes mencionadas.

A pensar de la bondad del ajuste en los tres casos para todas y cada una de las fuentes, existen ligeras diferencias entre ellas. La Log-normal y la Exponencial se adaptan muy bien a todas las distribuciones observadas, si bien, aunque no se detecte en el resumen que adjunto, la primera funciona mejor en grandes ámbitos, por ejemplo a nivel estatal, y la segunda la supera para estimar la distribución de ámbitos reducidos, como el municipal, en la que los rangos son más reducidos y los valores extremos aparecen mejor recogidos por la linealidad suave de la exponencial. Por su parte, la distribución de Pareto aparenta tener una menor calidad relativa de ajuste, si bien, los

\footnotetext{
${ }^{20}$ Para introducirse y consultar la teoría y aplicación general de los ajustes estadísticos, existen varios manuales que lo exponen, entre ellos: Sixto Ríos (Análisis estadístico aplicado, Paraninfo, Madrid, 1972); Calot (Curso de estadistica descriptiva, Paraninfo, Madrid, 1985); Grais (Méthodes statistiques, Dunod, París, 1974); Leboeuf, Roque y Guegand (Cours de probabilités et statistiques, Ellipses, París, 1991); Arnaiz Vellando (Introducción a la estadística teórica, Lex nova, Valladolid, 1978); Caron y Tassi (Problemes résolus de statistique mathématique, Economica, París, 1991)

${ }^{21}$ A lo largo de los últimos años, la investigación sobre las distribuciones probabilísticas en general y de la distribución de las rentas y los ingresos, en particular, se han centrado en la posibilidad de alcanzar una base teórica para las distribuciones de las rentas personales y un ratio explicativo entre la distribución funcional y personal de las rentas. Las contribuciones más importantes en el primer caso son las de Thurow (1970), Metcalf (1972), Beach (1976) y Dagum (1978). En el segundo caso, es preciso menciona a Lebergott (1964), Tuckman y Brosch (1974) y Dagum y Théorét (1978).
} 
índices de Gini teóricos son los más próximos a los observados, especialmente si los calculamos por el procedimiento de la versión de ley paretiana de segundo orden propuesta por M. Barbut, que excluye la necesidad de un mínimo inicial.

\section{Cuadro n. ${ }^{\circ} 4$ \\ Bondad del ajuste}

\begin{tabular}{|c|c|c|c|c|c|}
\hline \multicolumn{3}{|c|}{ Fuentes de datos observados } & \multicolumn{3}{|c|}{ Coeficiente de determinación } \\
\hline Tipo de Renta & Fuente & Año & Exponencial & Lognormal & Pareto \\
\hline $\begin{array}{l}\text { IRPF declarado } \\
\text { en Galicia }\end{array}$ & Agencia Tributaria & 1988 & 0,999 & 0,998 & 0,926 \\
\hline $\begin{array}{l}\text { Ingresos per cápita } \\
\text { España }\end{array}$ & $\begin{array}{l}\text { Encuesta de Presupuestos } \\
\text { Familiares (INE) }\end{array}$ & 1991 & 0,972 & 0,997 & 0,902 \\
\hline $\begin{array}{l}\text { Ingresos por hogar. } \\
\text { España }\end{array}$ & $\begin{array}{l}\text { Encuesta de Presupuestos } \\
\text { Familiares (INE) }\end{array}$ & 1991 & 0,970 & 0,975 & 0,823 \\
\hline $\begin{array}{l}\text { Ingresos por hogar. } \\
\text { España }\end{array}$ & Panel de hogares. (INE) & 1994 & 0,996 & 0,998 & 0,880 \\
\hline $\begin{array}{l}\text { Salarios } \\
\text { (asalariados). España }\end{array}$ & Agencia Tributaria & 1998 & 0,997 & 0,988 & 0,877 \\
\hline $\begin{array}{l}\text { Nivel de Renta } \\
\text { Municipal. España }\end{array}$ & La Caixa & 1997 & 0,942 & 0,996 & 0,888 \\
\hline $\begin{array}{l}\text { Ingresos por hogar. } \\
\text { Galicia }\end{array}$ & $\begin{array}{l}\text { Encuesta Condiciones de } \\
\text { Vida de los Hogares (IGE) }\end{array}$ & 1999 & 0,999 & 0.998 & 0,858 \\
\hline
\end{tabular}

Una explicación del por qué de tales diferencias se obtiene de inmediato al visualizar las gráficas de sus respectivas funciones de densidad. Las tres se asemejan a partir del valor modal. Es en los datos iniciales, los de la población con menor renta, lo que hace que una funcione mejor que otra. La Log-normal tiene una tendencia a la simetría, con un punto de partida inicial que recoge bien los valores inferiores. La de Pareto, si no se modifica, tiende a excederse en la sobrerrepresentación de las rentas más bajas y, finalmente, la Exponencial, es, a priori, una distribución que funciona bien en todos los casos, excepto en aquellas distribuciones con fuerte simetría.

En el Cuadro n. $^{\circ} 5$ se representa gráficamente uno de los casos estudiados, el de la distribución salarial declarada en la Agencia Tributaria española en 1998. Por restricciones legales, son escasos los declarantes por debajo del millón de pesetas anuales, con lo que el valor modal aparece como la más baja renta de las observadas. La ley exponencial es, por su parte la que más se aproxima a la 
observada, mientras que la de Pareto (en su versión de primera especie) es la que más se separa. La Log-normal, por su parte, se mantiene entre las anteriores, mostrando su tendencia a la inflexión entre los salarios más reducidos. Todas ellas sobrevaloran los valores inferiores e infravaloran, a su vez, los intervalos intermedios recogidos, para, finalmente, ajustar bien los efectivos con rentas salariales altas.

El Cuadro $n .^{\circ} 6$ muestra una distribución de la renta en los hogares gallegos derivada de los datos de la Encuesta sobre Condiciones de Vida de los Hogares, realizada por el Instituto Galego de Estatística en 1999. Estamos ante un ejemplo más global, en la que se recoge un mayor volumen de rentas bajas. Aparece, ahora sí, un origen en cero, con un rápido crecimiento hasta el valor modal, que se sitúa en las 125.000 pts. mensuales por hogar. El incremento de la renta reproduce paulatinamente un decrecimiento cada vez menos intenso de efectivos de hogares. La distribución exponencial, nuevamente, es la que más se aproxima a la observada, reproduciendo un sesgo de asimetría hacia las rentas más bajas, que se incrementará en los otros dos modelos. La ley paretiana, por su parte, es la que muestra un mayor alejamiento relativo de los datos observados, mientras que la Log-normal se sitúa entre ambos modelos teóricos.

\section{Cuadro n. ${ }^{\circ} 5$ \\ Representación de las funciones de densidad sobre salarios en España según fuentes tributarias en 1998}

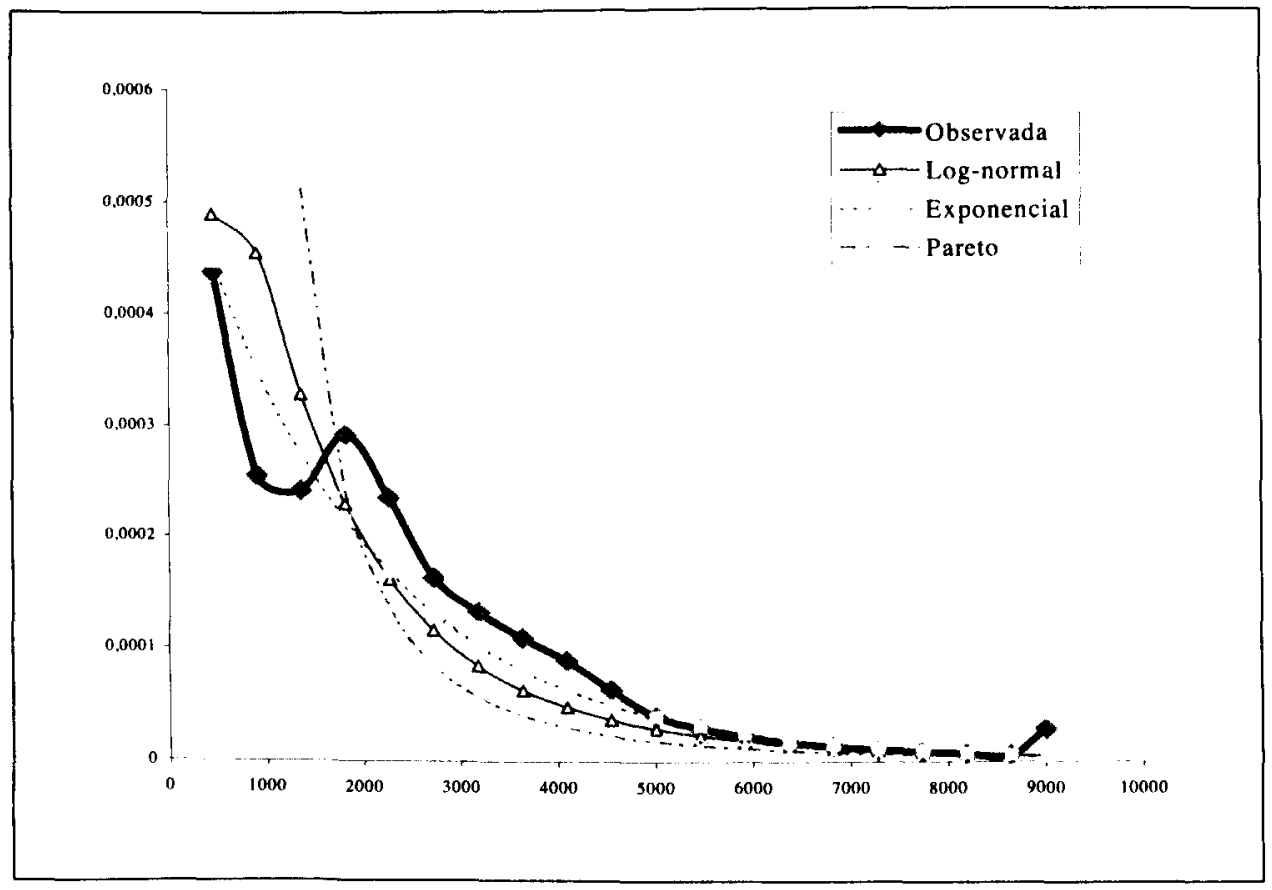


Cuadro n. ${ }^{\circ} 6$

Representación de las funciones de densidad sobre rentas en los hogares gallegos en 1999

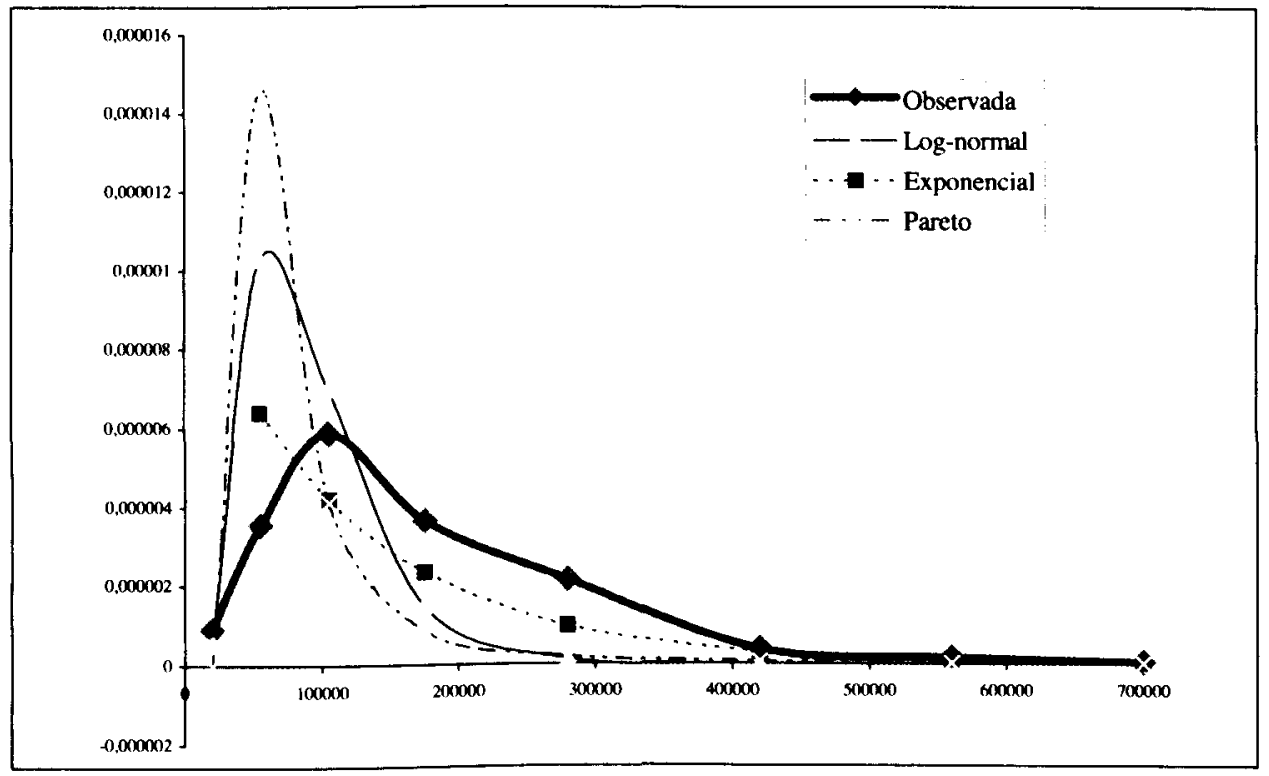

\section{ANEXO: CÁlCULO DEL PROCEDIMIENTO DE AJUSTE}

En este último apartado se definen cada una de las anteriores distribuciones y se expone, con un objetivo esencialmente pedagógico, el procedimiento de ajuste a los datos observados.

\subsection{La ley Log-normal}

Si suponemos que el logaritmo de una variable $x$ se distribuye normalmente, $x$ se distribuye siguiendo una ley Log-normal con una función de densidad:

$$
f(x)=\frac{1}{\sigma x \sqrt{2 \pi}} \cdot e^{-\frac{1}{2}\left(\frac{\log x-\mu}{\sigma}\right)^{2}} x \geq 0
$$

$\mu$ y $\sigma$ son la media y la varianza de $\log x$. $\mu$ es al mismo tiempo mediana de $x$ y de $\log x$. La curva representativa de $f(x)$ crece de cero hasta un valor modal alcanzado por un $x$ inferior a $\mu$, después decrece despacio y tiende a cero mientras $x$ tienda hacia infinito. Su función de distribución puede escribirse:

$$
F(x)=P(X \leq x)=P(\log X \leq \log x)
$$


Para ver si una distribución observada sigue una ley log-normal podemos proceder por dos métodos ${ }^{22}$ : el método gráfico y el ajuste lineal. El primero consiste en representar los valores observados en una papel graduado con una escala gaussiana en ordenadas y una escala logarítmica en abcisas (gradación gaussologarítmica). Si los valores adoptan la forma de una recta (la recta de Henri), la distribución observada es tipo log-normal. Este método evita calcular los logaritmos al permitirnos trabajar con los valores observados.

Para obtener un ajuste por linearización, examinaremos la proximidad de los valores observados a una recta en la cual la variable independiente es logarítmica. La función de distribución puede escribirse en función de las áreas de una distribución normal $(0,1)$. Llamemos $U_{i}$ a cada área $\mathrm{N}(0,1)$ de $\mathrm{F}(\mathrm{x})$. Tendremos entonces

$$
U_{i}=a+b \log x_{i}
$$

Por otra parte sabemos que las áreas de las probabilidades de una distribución normal $N(0,1)$ van a ser

$$
U_{i}=\frac{\log x_{i}-\mu}{\sigma}
$$

de donde

$$
\pm \frac{\mu}{\sigma}+\frac{1}{\sigma} \log x_{i}=a+b \log x_{i}
$$

De la misma fórmula de la cual nos hemos servido para obtener la recta de regresión, podemos servirnos para calcular los parámetros $(\mu, \sigma) \mathrm{y}$, resolviendo la ecuación anterior, obtenemos:

$$
\mu=-\frac{a}{b} ; \sigma=\frac{1}{b}
$$

Una vez obtenidos estos valores, podemos calcular la distribución teórica resultante, así como los parámetros media, mediana y mínimo. Finalmente, el índice teórico de Gini nos proporcionará una idea de la concentración de los valores, midiendo de esta forma la desigualdad existente en la distribución. ya que

La media teórica se obtiene de esta manera, a partir de los valores de $\mu$ y $\sigma$,

$$
E(x)=\int_{0}^{\infty} x f(x) d x=\frac{1}{\sigma \sqrt{2 \pi}} \int_{0}^{\infty} e^{-\frac{1}{2 \sigma^{2}}(\log x-\mu)^{2}} d x=e^{-\mu+\frac{\sigma^{2}}{2}}
$$

${ }^{22}$ Siguiendo a Aitchenson y Brown (1963). 
La mediana $M_{e}$ es un valor tal que $F\left(M_{e}\right)=1 / 2$. Ahora bien, como he mostrado anteriormente, $F(x)=\phi_{1}(\log x)$, donde $\phi$ es la función de distribución de una $N(\mu, \sigma)$. Así pues $F\left(M_{e}\right)=\phi_{1}\left(\log M_{e}\right)=1 / 2 \Leftrightarrow \log M_{e}=\mu$ ya que en una distribución normal la mediana coincide con la media. En definitiva, $M_{e}=e^{\mu}$.

Podemos trabajar en distribuciones sin mínimo preestablecido, lo que se convierte en una de sus características principales. Finalmente, el índice teórico de Gini de obtiene resolviendo:

$$
G=\frac{(1 \pm 2 \Phi)\left( \pm \frac{\sigma}{\sqrt{2}}\right) e^{\mu+\frac{\sigma^{2}}{2}}}{X_{0}+e^{\mu+\frac{\sigma^{2}}{2}}}
$$

\subsection{Ley exponencial y su familia}

La ley exponencial tiene una función de densidad: $\mathrm{f}(x)=\lambda e^{-\lambda\left(x-X_{0}\right)}$; para $x \geq 0$. La función de distribución es: $F(x)=1-e^{-\lambda\left(x-x_{0}\right)} \quad \forall x \geq x_{0}$ siendo $\lambda \geq$ 0 ; o, lo que es lo mismo, $1-F(x)=e^{-\lambda\left(x-x_{0}\right)}$.

Para ver si una distribución observada es de tipo exponencial podemos examinar la distribución de los datos observados en papel semilogarítmico y, si los puntos $\left(x_{i}, \ln \left(1-F\left(x_{i}\right)\right)\right)$ se sitúan aproximadamente en una recta, el modelo teórico es válido.

Si ajustamos por linearización, con los puntos $\left(x_{i}, \ln \left(1-F\left(x_{i}\right)\right)\right)$ se buscan los valores $a$ y $b$ de la recta: $\ln (1-F(x))=a+b x=-\lambda\left(x-x_{0}\right)=\lambda \mathrm{x}_{0}-\lambda \mathrm{x}$.

Los parámetros $\lambda$ y $\mathrm{x}_{n}$ se obtienen a partir de los coeficientes de la recta de regresión, ya que $a+b x=\lambda x_{0}-\lambda x \Rightarrow \lambda=-b$ y $\mathrm{X}_{0}=-\frac{a}{b}$ con lo que hemos obtenido el mínimo de la distribución como relación pro cociente entre $a$ y $b$.

Si concluimos que nuestros datos observados siguen aproximadamente una distribución exponencial, su distribución será, aproximadamente del tipo: 


\section{Cuadro n. ${ }^{\circ} 7$ \\ Distribución exponencial}

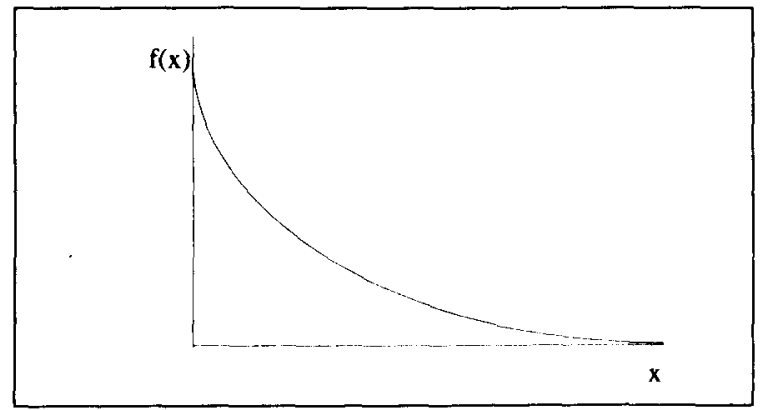

La media de la distribución exponencial es: $E(x)=\int_{x_{0}}^{\infty} x \lambda e^{-\lambda\left(x-x_{0}\right)} d x=\frac{1}{\lambda}+X_{0}$.

La mediana es: $F\left(M_{e}\right)=1-e^{-\lambda\left(M_{e}-X_{0}\right)}=X_{0}-\frac{1}{\lambda} \ln \frac{1}{2}$. Finalmente, el indice de Gini se obtiene resolviendo: $\frac{1}{2} \frac{1}{1+\lambda X_{0}}$.

\subsection{Las Leyes Paretianas}

\subsubsection{Planteamiento general}

La distribución de Pareto estuvo pensada desde su concepción para el describir la distribución de la renta en la población. Pareto observó que a partir de un cierto valor de $\mathrm{x}$, el número de individuos que tienen unos ingresos superiores a $x$ decrece proporcionalmente a $1 / x^{\alpha}$. Decimos que ley pertenece a la familia de las leyes paretianas cuando cumple la propiedad anterior. $\alpha$ es normalmente conocido con el nombre de coeficiente de Pareto. En la mayor parte de los casos el coeficiente de Pareto $\alpha$ es inferior a 2, lo que implica que la ley no tenga varianza finita ${ }^{23}$.

Desde un principio Pareto formuló varios modelos: La ley de Pareto de primera especie tendrá como Función de Distribución:

$$
F(x)=1-\left(\frac{X_{0}}{x}\right)^{\alpha} ; \mathrm{x} \geq \mathrm{X}_{0} \geq 0
$$

${ }^{23}$ Esta observación fue hecha distintas épocas y países. Véanse especialmente los trabajos de Champernowne (1952); Davis (1941), Hayakawa (1951)y Renyi (1966). 
Ley de Pareto de segunda especie (que se convierte en más precisa por la inclusión de un segundo parámetro $c$ ), responde a la siguiente Función de Distribución:

$$
F(x)=1-\left(\frac{X_{0}}{x+c}\right)^{\alpha} ; \mathrm{x} \geq \mathrm{X}_{0} \geq 0
$$

La ley de Pareto de tercera especie, aunque no pueda considerarse estrictamente paretiana, sí lo es en la práctica por tratarse de valores de $b$ muy pequeños:

$$
F(x)=1-\frac{A e^{ \pm b x}}{(x+c)^{\alpha}} ; \forall x \geq 0
$$

Las sucesivas correcciones que se van señalando, al incluir un mayor número de parámetros en cada nueva ley, incide en moldear la distribución con un efecto que la aleja cada vez más de la exponencial y genera una forma peculiar, entre aquella y la Log-normal, que la convierte en la distribución con mayor capacidad explicativa, pero también con mayor dificultad de cálculo y ajuste. Se consigue, así, una distribución truncada, ya que, las distribuciones observadas, no decrecen aproximativamente como una función potencial más que a partir de un cierto umbral (Lecrerc, 1972:29) lo que conlleva una búsqueda de las leyes que se ajusten bien al conjunto de la distribución.

\section{Cuadro no 8 \\ Curva de una distribución paretiana de segunda especie}

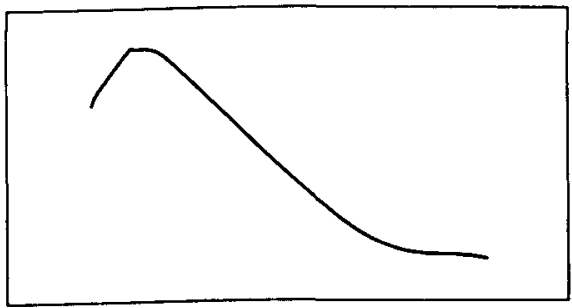

La mencionada capacidad explicativa de la ley de segunda especie se ve limitada por la imposibilidad práctica de diseñar un procedimiento estadístico de ajuste, aunque existe una solución empírica para calcular los parámetros derivados de la misma, que posteriormente expondré, y que en su día fueron desarrollados por Marc Barbut. Con todo, la ley de primera especie permite una verificación suficiente sobre la bondad del ajuste, tal como vimos en el Cuadro $\mathrm{n}^{\circ} 5$ y en base a la lógica que sigue a continuación.

La función de densidad de una ley de Pareto de primera especie puede formularse como: $f(x)=\frac{\alpha}{X_{0}}\left(\frac{X_{0}}{x}\right)^{\alpha}$, en donde $\mathrm{X}_{0}$ es el mínimo de la distribución 
y, por lo tanto, deberá ser igual o inferior a cada valor de las $x$, además de positivo. Su correspondiente Función de distribución es $F(x)=1-(\lambda / x)^{\alpha}$ y su opuesta será pues $1-F(x)=1-(\lambda / x)^{\alpha}$. Para ajustar la distribución observada a la teórica debemos transformar esta ecuación en logarítmica, obteniendo así la función: $\log (1 \pm F(x))=\alpha(\log \lambda \pm \log x)$.

Con el cálculo de los valores de la ecuación anterior podemos estimar una recta, $\log y=a+b \log x$ por el método de los mínimos cuadrados. Si se representan los valores observados $\left(\log x_{i}, \log \left(1-H_{i}\right)\right)$ sobre un papel doblemente logarítmico y si estos se ajustan bien a una ley paretiana, obtendríamos una recta de pendiente negativa. Es decir, cuando la distribución observada está próxima de la de una distribución de Pareto, los puntos de coordenadas $\left(\log x_{i}, \log \left(1-H_{i}\right)\right)$ deben situarse próximos a una recta. Se puede calcular también un coeficiente de correlación para verificar la calidad del ajuste.

El cálculo de la media teórica es obtenido por $\frac{\alpha}{\alpha-1} X_{0}$, y existirá si y sólo si $\alpha>1$. La mediana teórica paretiana se obtine como: $M_{e}=2^{1 / \alpha}$. El mínimo de la distribución es: $X_{0}=10^{-a / \alpha}$, siendo $a$ el valor obtenido en la recta de regresión, como $a=\bar{y}-b \bar{x}$. Finalmente, obtendremos el índice teórico de Gini resolviendo: $G=\frac{1}{2 \alpha-1}$.

\subsubsection{El método de cálculo propuesto por M. Barbut}

En un artículo aparecido en 1988 en la revista Histoire \& Mesure ${ }^{24}$, M. Barbut, con la aparente finalidad de criticar otro artículo anteriormente aparecido en la misma revista de Denis Morsa ${ }^{25}$, lleva a cabo un cálculo empírico de los parámetros de una ley paretiana con otra metodología diferente a la expuesta de linearización por logaritmos ${ }^{26}$.

Sea una variable numérica de función de distribución $F(x)$ para $x \geq a>0$. Sea, también, $F(x)=1-P(x)$ la proporción de valores de la variable que son inferiores o iguales a $\mathrm{x}, \mathrm{y}$, finalmente, para cada $x$, sea $M(x)$, el valor medio de los valores de esta variable que son superiores o iguales a $x$; en fórmulas:

\footnotetext{
${ }^{24}$ Barbut (1988).

${ }^{25}$ Morsa (1987).

${ }^{26}$ Esta no es la primera ocasión en la que Barbut explica esta metodología. Los aspectos más teóricos pueden consultarse en Barbut (1989).
} 


$$
\forall x \geq a, M(x)=\frac{1}{1-F(x)} \int_{0}^{\infty} t d F(t)=\frac{1}{P(x)} \int_{0}^{\infty} t d F(t)
$$

Entonces la distribución de esta variable es paretiana si y solamente si ${ }^{27}$ :

$$
\begin{aligned}
& \left(P(x)=\left(\frac{a}{x}\right)^{\alpha}, \alpha>1\right) \\
& M(x)=B x .
\end{aligned}
$$

donde el coeficiente $\beta$ aparece ligado al exponente $\alpha$ por la relación:

$$
\frac{1}{\alpha}+\frac{1}{\beta}=1 \quad \mathrm{y} \text { así, } \beta=\frac{\alpha}{\alpha-1}
$$

El $M(x)$ se obtiene empíricamente dividiendo la cantidad acumulada en el rango $x$, por el número de individuos a los que concierne. Podemos obtener pues una media $M(x)$ para cada uno de los intervalos. Si dividimos esta media por el número de individuos de cada intervalo obtenemos un ratio ${ }^{28} M(x) / x$ que nos servirá para estimar el valor del parámetro $\beta$, ya que $M(x)=B x \Rightarrow M(x) / x=\beta$.

Podemos calcular también el coeficiente de correlación entre los puntos $\left(x_{i}, M\left(x_{i}\right)\right)$ para verificar la calidad del ajuste. Este coeficiente se calcula sobre las observaciones mismas y no sobre sus logaritmos.

El valor de $\beta$ puede estimarse por la media aritmética de los $M\left(x_{i}\right) / x_{i}$ observados. Se puede también escoger el valor $\beta$ que minimice la suma de las desviaciones absolutas entre el modelo y las observaciones:

$$
\sum\left|\varepsilon_{i}\right|=\sum\left|M(x)-\beta x_{i}\right|
$$

También podemos obtener con facilidad el valor de $\bar{M}$, ya que es el valor medio de los valores observados en la población. A partir de ahí podemos calcular a y $\alpha$, ya que:

$$
a=\frac{\bar{M}}{\beta} \quad \text { y } \quad \alpha=\frac{\beta}{\beta-1}
$$

\footnotetext{
${ }^{27}$ En el caso de la ley del primer tipo.

${ }^{28}$ Si la distribución sigue una ley de Pareto del primer tipo, los ratios obtenidos deben ser aproximadamente constantes.
} 
Finalmente estimamos la distribución teórica por medio de:

$$
P=\left(\frac{a}{\beta}\right)^{\alpha}, x \geq a
$$

\subsubsection{Ley del segundo tipo}

Cuando las $x_{i}$ crecen y los ratios $M(x) / x$ decrecen, Barbut propone una Ley de Pareto del Segundo tipo:

$$
\frac{M(x)}{x}=\frac{\beta x+\mu}{x}=\beta+\frac{\mu}{x}
$$

$\beta$ у $\mu$ serán estimadas por el método de los mínimos cuadrados:

$$
M(x)=\beta x+\mu+\varepsilon(x)
$$

Así obtenemos: $a=\frac{\bar{M}-\mu}{\beta}$ y $c=\frac{\mu}{\beta-1}$

Las probabilidades teóricas se obtienen por: $P(x)=\left(\frac{a+c}{x+c}\right)^{\alpha}$

El índice de Gini se resolvería calculando $G=\frac{B=1}{B+1}\left(1+\frac{c}{M}\right)$ y, finalmente, la mediana, por: $M_{e}=2^{1 / \alpha}(a+c) \pm c$ 
MODELIZACIÓN DEL ANÁLISIS DE LAS DESIGUALDADES EN LA DISTRIBUCIÓN...
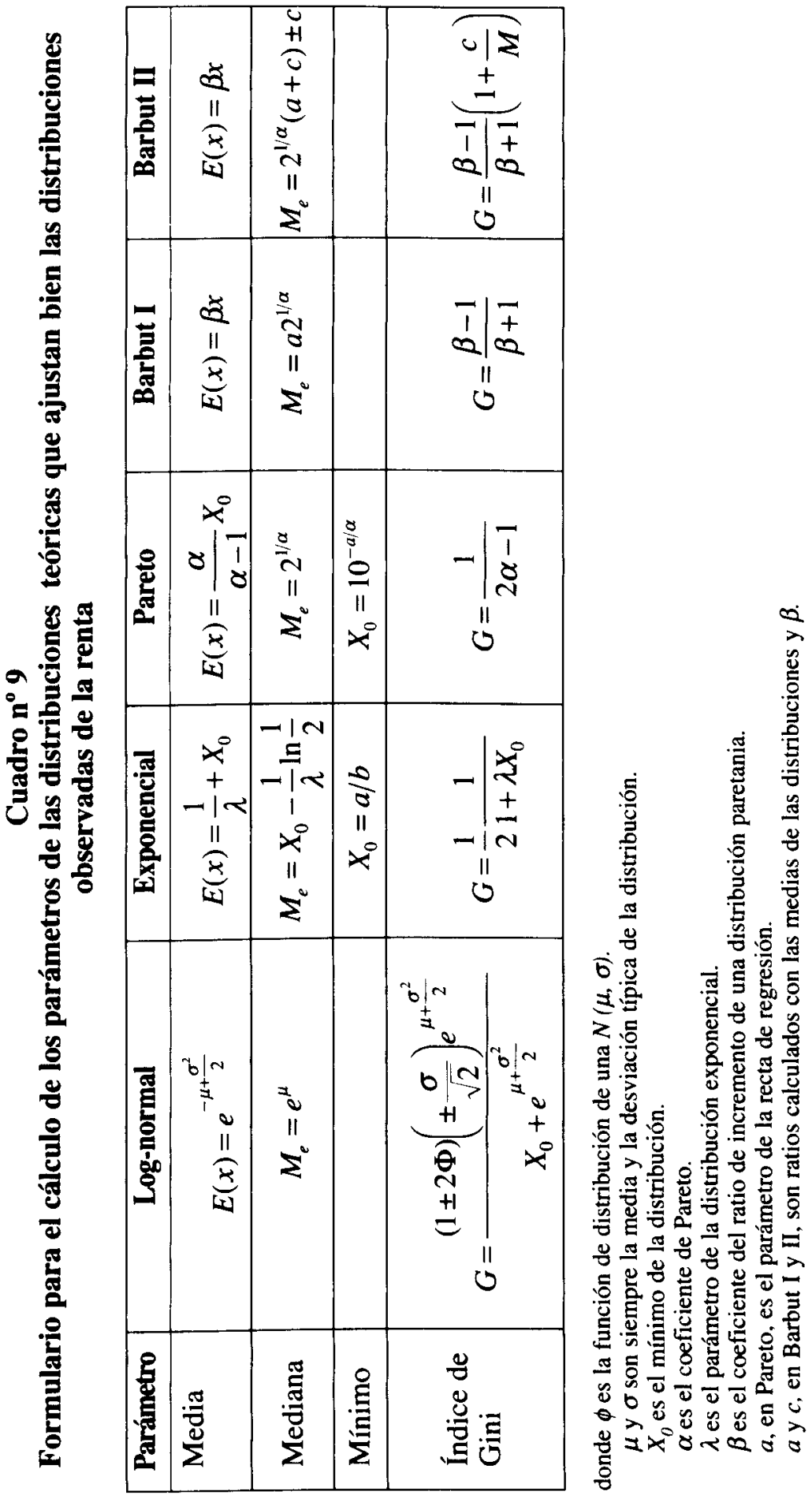


\section{BIBLIOGRAFIA DE REFERENCIA}

Aitcheson, J. y Brown, J.A.C. (1963): The Lognormal distribution, Cambridge University Press, Cambridge.

AtKinson, A.B. (1981): La economía de la desigualdad, Barcelona, Editorial Crítica.. - (1983): «On the Measurement of Inequality», Journal of Economic Theory, Vol. 2, pp. 244-263.

Amorós, C. (1994): «Igualdad e identidad» en El concepto de igualdad de Amelia Valcárcel (Comp.), Madrid: Editorial Pablo Iglesias, pp. 29-48.

BARBUT, M. (1988): «Des bons et des moins bons usages des distributions parétiennes en analyse des données», Histoire \& Mesure, Vol. III-1, pp. 111-128.

- (1989): «Distributions de type Parétien et représentation des inégalités», Mathématiques, Informatique et Sciences Humaines, n. ${ }^{\circ}$ 106, pp. 53-69.

BeACH, Ch. (1976): «Cyclical Impacts on the Personal Distribution of Income», Economic and Social measurement, Vol. $5, \mathrm{n}^{\circ} 1$, pp. 29-52.

Carling, Alan. H. (1991): Social Division, Londres: Verso.

Crompton, R. (1993): Clase y estratificación. Una introducción a los debates actuales, Madrid, Tecnos, 1994.

Champernowne, D. G. (1952): «The graduation of income distributions», Econometrica, 20, pp. 591-615.

DaGum, C. (1978): «Toward a General Model of Prodution and Distribution Models», Hommage à François Perroux, Presses Universitaires de Grenoble, pp. 413-436.

DAGUM, C. y THEORET, C. (1978): «The Impact of the Composition of Income Upon the Size Distribution of Income», Studi in Onore di G. Demaria, Padova, CEDAM, pp. 907-926.

DAvis, H.T. (1941): The analysis of economic time-series, Cowles Comission Monograph, n. ${ }^{\circ}$ 6, Bloomington, The Principia Press.

Garrido, L. J. y Toharia, L. (1996): «Paro y desigualdad», en VV.AA., Pobreza, necesidad y discriminación, Madrid, Fundación Argentaria-Visor, pp. 9-64.

HayaKaWA, M. (1951): "The application of Pareto's law of income to japanese data», Econometrica, 19, pp. 174-183.

I.N.E. (1989): El estudio de la pobreza en España, Madrid, Instituto Nacional de Estadística.

KaELBLe, H. (1994): Desigualdad y movilidad social en los siglos XIX y XX, Madrid, Ministerio de Trabajo y Seguridad Social.

LeberGotT, S., (1964); «Factor Shares in the Long Terme: Some Theoretical and Statistical Aspects», in Studies in Income and Wealth, Princeton University Press, Vol. 27, pp. 53-86.

LECREC, B. (1972): «Distributions statistiques et lois de probabilité», Cahiers mathématiques,.$^{\circ} 4$, (número monográfico) París, Mouton/Gautier-Villars.

MerCader, M. (1993): «Bajos niveles de renta en España y una comparación con el Reino Unido y Francia», I Simposio sobre Igualdad y Distribución de la Renta y de la Riqueza, multicopiado, Madrid, Fundación Argentaria.

MetCaF, Ch. (1972): An Econometric Model of the Income Distribution, Chicago, Markham Publishing Company.

MORSA, D. (1987). «Distribution de la population des villes et des localités de la Principauté de Liège à la fin du XVIIIe siècle», Histoire \& Mesure, Vol. II-2, pp. 73-92.

RAE, D. (1981): Equalities, Cambridge, Harvard University Press.

Rawls, J. (1979): Teoría de la Justicia, Madrid, F.C.E..

RENYI, A. (1966): Calcul des probabilités avec un appendice sur la théorie de l'information, París, Dunod. 
SEN, A.K. (1973), On economic ineqality, Oxford, Oxford University Press. - (1995): Nuevo examen de la desigualdad, Madrid, Alianza Editorial.

THEIL, H. (1967): Economics and Information Theory, Amsterdam, North-Holland Publishing Company.

Thurow, L. (1970): «Analyzing the American Income Distribution», American Economic Review, Vol. 48, pp. 261-269.

Tuckman, H.; Brosch, G. (1974): «Changes in Personal Income and Their Effect on Income Shares», Southern Economic Journal, pp. 78-96.

ValĆ́rCel, A. (Comp.) (1994): El concepto de Igualdad, Madrid, Editorial Pablo Iglesias.

\title{
RESUMEN
}

Partiendo del marco teórico del análisis de las desigualdades sociales, es posible distinguir tres tipos de metodologías analíticas sobre las situaciones susceptibles de ser consideradas como desigualitarias. Entre ellas, las referidas a la Distribución de la Renta, forman parte de uno de esos tipos. Este artículo presenta los aspectos fundamentales de ese marco teórico, así como la tipología mencionada, y se adentra en las características e indicadores habituales para medir el grado y la intensidad de las desigualdades observables en el reparto de la renta total de un conjunto poblacional. Se muestra, así mismo, como tales distribuciones siguen un modelo que viene bien representado por una serie de leyes probabilísticas, tales como la Log-normal, la Exponencial y la de Pareto. La bondad de ajuste de estos modelos teóricos se verifica empíricamente analizando siete distribuciones diferentes, en las que, en todos los casos, se obtienen coeficientes de correlación superiores al $80 \%$.

\begin{abstract}
Starting from the theoretical framework of the analysis of social inequalities, three types of analytical methodologies for situations likely to be considered of an unequal nature can be distinguished. Among such situations, those concerning Income Distribution are one of these types. This paper presents the fundamental aspects of the theoretical framework, as well as the aforementioned typology and takes a close look at the common characteristics and indicators in order to measure the degree and intensity of the inequalities which can be observed in the distribution of the total income of a population group. The paper also shows the way in which such distributions follow a model represented by a series of probability laws, such as Log-normal, Exponential and Pareto. The goodness of fit of these theoretical models can be verified empirically by analysing seven different distributions in which, in all cases, correlation coefficients higher than $80 \%$ have been obtained.
\end{abstract}

